\title{
Derivation of a Multiparameter Gamma Model for Analyzing the Residence-Time Distribution Function for Nonideal Flow Systems as an Alternative to the Advection-Dispersion Equation
}

\author{
Irucka Embry, ${ }_{1}^{1}$ Victor Roland, ${ }^{1}$ Oluropo Agbaje, ${ }^{1}$ Valetta Watson,, Marquan Martin, \\ Roger Painter, ${ }^{1}$ Tom Byl, ${ }^{2}$ and Lonnie Sharpe ${ }^{3}$ \\ ${ }^{1}$ Department of Civil \& Environmental Engineering, Tennessee State University (TSU), Nashville, TN 37209, USA \\ ${ }^{2}$ United States Geological Survey (USGS), Nashville, TN 37211, USA \\ ${ }^{3}$ Massie Chair of Excellence, Tennessee State University (TSU), Nashville, TN 37209, USA \\ Correspondence should be addressed to Irucka Embry; iruckae@mail2world.com
}

Received 27 September 2012; Accepted 19 October 2012

Academic Editors: D. A. Drew and J. A. A. González

Copyright ( $\odot 2013$ Irucka Embry et al. This is an open access article distributed under the Creative Commons Attribution License, which permits unrestricted use, distribution, and reproduction in any medium, provided the original work is properly cited.

\begin{abstract}
A new residence-time distribution (RTD) function has been developed and applied to quantitative dye studies as an alternative to the traditional advection-dispersion equation (AdDE). The new method is based on a jointly combined four-parameter gamma probability density function (PDF). The gamma residence-time distribution (RTD) function and its first and second moments are derived from the individual two-parameter gamma distributions of randomly distributed variables, tracer travel distance, and linear velocity, which are based on their relationship with time. The gamma RTD function was used on a steady-state, nonideal system modeled as a plug-flow reactor (PFR) in the laboratory to validate the effectiveness of the model. The normalized forms of the gamma RTD and the advection-dispersion equation RTD were compared with the normalized tracer RTD. The normalized gamma RTD had a lower mean-absolute deviation (MAD) (0.16) than the normalized form of the advection-dispersion equation (0.26) when compared to the normalized tracer RTD. The gamma RTD function is tied back to the actual physical site due to its randomly distributed variables. The results validate using the gamma RTD as a suitable alternative to the advection-dispersion equation for quantitative tracer studies of non-ideal flow systems.
\end{abstract}

\section{Introduction}

Researchers have used the distribution of residence times to examine the characteristics of a nonideal flow reactor or system. The residence-time distribution (RTD) was first proposed to analyze chemical reactor performance in a paper by MacMullin and Weber in 1935 [1-3]. Only after Danckwerts' publication of "Continuous flow systems. Distribution of residence times," in 1953, was the RTD theory organized in a more structured manner and most of the distributions were classified [2-5]. Many people still use Danckwerts' work as their foundation for analysis of systems with the RTD model. The residence-time distribution of a system characterizes the mixing that happens in a system. The residence-time distribution function is quantified by the term $E(t) . E(t)$ describes quantitatively the amount of time that different fluid particles have spent in the system. $E(t)$ is also a probability density function (PDF) that defines the probability that a particle entering the system will remain there for a time $t$ (see [1-8] for a thorough explanation of the background theory to mixing and RTD). Equation (1) is generally used to determine the RTD function $[2,7]$ as

$$
E(t)=\frac{C(t)}{\int_{0}^{\infty} C(t) d t},
$$

where $C(t)$ is the concentration of the tracer over time and the plot of concentration versus time is the tracer breakthrough curve. 
It is important to note that all molecules will eventually leave the system (this is also a method used to normalize the distribution) $[2,3,8]$, thus

$$
\int_{0}^{\infty} E(t) d t=1
$$

Two important parameters derived from the RTD function are the mean residence time $\left(t_{m}\right)[2,7]$ as

$$
t_{m}=\frac{\int_{0}^{\infty} t E(t) d t}{\int_{0}^{\infty} E(t) d t}=\int_{0}^{\infty} t E(t) d t
$$

and the first moment about the mean of the RTD function (distribution variance or $\sigma^{2}$ ) [2] as

$$
\sigma^{2}=\int_{0}^{\infty}\left(t-t_{m}\right)^{2} E(t) d t .
$$

Although the RTD was originally applied to designing chemical reactors, the RTD has been used in a variety of other applications (see [1-28] and their references for both a thorough discussion of the RTD and its widespread applications). The RTD has also been referred to in the literature as the detention-time distribution (DTD) [29], transit-time distribution (TTD) [30], travel-time distribution [31-33], and hydraulic residence-time distribution (HRTD) [34, 35]. Some researchers concentrate their efforts on obtaining the parameters derived from the RTD function to characterize the flow patterns that they are analyzing $[36,37]$.

Generally, the main model used to describe the residencetime distribution of a system has been the one-parameter advection-dispersion equation or model (AdDE) [7, 19, 21$28,31,33$ ]. In the literature, the AdDE has also been called the axial dispersion or diffusion equation or model (AxDE) or (ADM) $[2,3,5,6,8-10,13-15,18]$; the advection diffusion equation or "diffusion with bulk flow equation" [10]; or the convection dispersion or diffusion equation (CDE) [18, $38,39]$. The advection-dispersion equation exhibits an RTD function curve which can appear Gaussian based on the conditions [19]. The AdDE model with its single parameter and Gaussian-shaped curves is inadequate for visualizing the nonideal flow RTD $[9,19,27,28]$. Also, it is true that the symmetric AdDE Gaussian-shaped curve predicts a finite tracer concentration at time $=0$, but this is not true for the AdDE solution at that time. Lastly, the Gaussianshaped curve of the advection-dispersion equation does not adequately display the fullness of tracer breakthrough curves that generally have long upper tails [27]. Thus, we decided to derive an RTD function for non-ideal flow systems by combining two, two-parameter gamma distributions. The gamma distribution resembles many natural processes and has been used widely in complex applications, thus it is a good model for non-ideal flow systems (see [29-33, 40-51] for both a thorough discussion of the gamma distribution and its various applications).

This jointly combined four-parameter gamma model allows for more flexibility to account for the nonlinear aspects $[30,31]$ of a non-ideal flow system than the single parameter AdDE model; however, the gamma distribution's two parameters $(\alpha, \beta)$ do not have a clearly associated physical interpretation [30] as does the AdDE model with the Péclet number. To address this issue, the gamma distribution for the RTD was derived based on the assumption that the tracer travel distance and linear velocity of the system were gamma-distributed random variables. This assumption solves the problems regarding the physical interpretation as $\alpha_{1} \beta_{1}$ is associated with the mean travel distance of the tracer molecules while $\left(\alpha_{2}-1\right) \beta_{2}$ is associated with the mean travel linear velocity (mean travel distance/mean time in the system). Thus we assume that the solute moves with the water. We also assume that the ratio $\alpha_{1} \beta_{1} /\left(\alpha_{2}-1\right) \beta_{2}$ is approximately equal to the mean residence time or the mean time in the system $\left(t_{m}\right)$. The resulting four-parameter model is robust and better able to fit the normalized tracer RTD curve. In addition, the model parameters' relation to the linear velocity and the travel distance of the actual system simplifies the parameterization of the model by reducing the degrees of freedom from four to two.

Regarding non-ideal flow systems, we are assuming the system is isothermal and homogeneous and that the volume changes during the tracer study are assumed to be negligible $[5,6]$. We are also assuming that the time domain is steady state rather than transient. The authors in $[2,5-8]$ provide a thorough explanation of non-ideal flow systems.

\section{Derivation of the Four-Parameter Gamma Distribution RTD Model}

We are assuming that the residence time of tracer particles is similar to travel times of discrete water molecules in a nonideal flow system along flow paths. The flow paths for discrete water particles vary in length, local hydraulic gradient, and cross-section. Tracer sample concentration as a measure of the tracer flux at a given time is randomly distributed, but the approach developed in this paper does not apply a residencetime distribution directly to the concentration data. Instead, the arrival of molecules at the sampling point at a particular time is seen as a random event dependent on the distance traveled and speed of travel. Thus, the relation between travel distance and velocity reflected in the space time $(\tau)$ for a nonideal flow system as follows:

$$
\tau=\frac{V_{\mathrm{eq}}}{\mathrm{Q}}=\frac{\bar{L} A_{\mathrm{eq}}}{\bar{v} A_{\mathrm{eq}}}=\frac{\bar{L}}{\bar{v}},
$$

where both $L$ and $v$ represent independent random variables.

For modeling non-ideal flow systems, addressing the interaction of $L$ and $v$ is important because their independent values relate directly to important characteristics of the system. Specifically, those important characteristics are the following: distance traveled and the straight-line distance between the injection and sampling point(s), localized hydraulic gradient(s), and flow cross-section(s) along the flow path. For this reason, describing the tracer breakthrough curve in terms of a distribution derived from the joint PDF for $L$ and $v$ should provide better insight regarding the RTD for a non-ideal flow system. 
The literature suggests that the gamma distribution does well in describing tracer breakthrough curves for non-ideal flow systems [27-33]. The gamma distribution, which is frequently used as a probability model for waiting times, seems to adequately reflect the "long tail to the right" often observed in tracer breakthrough curves [22]. Based on this observation we assumed that $L$ and $v$ are independent random variables (irv) that have gamma PDFs as follows:

$$
f_{L}\left(x_{1}\right)=\frac{x_{1}^{\alpha_{1}-1} e^{-x_{1} / \beta_{1}}}{\Gamma\left(\alpha_{1}\right) \beta_{1}^{\alpha_{1}}} ; \quad \alpha_{1} \geq 1 ; \beta_{1} \geq 0, x_{1} \geq 0,
$$

where $\alpha_{1}$ and $\beta_{1}$ are the shape and scale parameters of the two-parameter gamma distribution, respectively, and $\Gamma(\alpha)$ is the gamma function $[40-45]$ as

$$
\begin{gathered}
\Gamma(\alpha)=\int_{0}^{\infty} x^{\alpha-1} e^{-x} d x, \\
f_{v}\left(x_{2}\right)=\frac{x_{2}^{\alpha_{2}-1} e^{-x_{2} / \beta_{2}}}{\Gamma\left(\alpha_{2}\right) \beta_{2}^{\alpha_{2}}} ; \quad \alpha_{2} \geq 1 ; \beta_{2} \geq 0, x_{2} \geq 0,
\end{gathered}
$$

where $\alpha_{2}$ and $\beta_{2}$ are the shape and scale parameters of the two-parameter gamma distribution, respectively and $\Gamma(\alpha)$ is the gamma function [40-45].

$$
f(x)=\frac{x^{\theta-1} e^{-x / \varphi}}{\varphi^{\theta} \Gamma(\theta)} ; \quad x>0 ; \theta, \varphi>0,
$$

this is the general formula for a two-parameter gamma distribution where $\theta$ and $\varphi$ are the shape and scale parameters of the distribution, respectively, and $\Gamma(\theta)$ is the gamma function [52].

The following mathematical discussion and (9)-(15) are from [52] "The distribution of residence time $(t)$ is derived from the Mellin convolution of the distribution of quotients of random variables where the PDF of the quotient

$$
Y=\frac{X_{1}}{X_{2}}=\left(X_{1}\right)\left(\frac{1}{X_{2}}\right),
$$

where $Y=t$ and $X_{1}=L$ and $X_{2}=v$ of two nonnegative irv's with PDFs $f_{L}\left(x_{1}\right)$ and $f_{v}\left(x_{2}\right)$ is expressible as the Mellin convolution

$$
h_{2}(y)=\int_{0}^{\infty} x_{2} f_{L}\left(y x_{2}\right) f_{v}\left(x_{2}\right) d x_{2},
$$

of $f_{L}\left(x_{1}\right)$ and $g_{2}\left(1 / x_{2}\right)$. This is established by utilizing a transformation

$$
Y=\frac{X_{1}}{X_{2}}, \quad X_{2}=X_{2},
$$

the inverse of which is

$$
X_{1}=Y X_{2}, \quad X_{2}=X_{2} .
$$

As the Jacobian of the transformation of (12) is

$$
J=\left|\begin{array}{ll}
\frac{\partial x_{1}}{\partial y} & \frac{\partial x_{1}}{\partial x_{2}} \\
\frac{\partial x_{2}}{\partial y} & \frac{\partial x_{2}}{\partial x_{2}}
\end{array}\right|=\left|\begin{array}{cc}
x_{2} & y \\
0 & 1
\end{array}\right|=x_{2},
$$

the joint PDF $f\left(x_{1}, x_{2}\right)=f_{L}\left(x_{1}\right) f_{v}\left(x_{2}\right)$ is transformed into $g\left(y, x_{2}\right)$, where

$$
g\left(y, x_{2}\right)=f_{L}\left(y x_{2}\right) f_{v}\left(x_{2}\right)|J|=x_{2} f_{L}\left(y x_{2}\right) f_{v}\left(x_{2}\right) .
$$

On integrating (14) with respect to $x_{2}$, one obtains the Mellin convolution \{in our case the PDF for the residence time $(t)$ is given by the marginal probability in (15)\}

$$
h_{2}(y)=\int_{0}^{\infty} g\left(y, x_{2}\right) d x_{2}=\int_{0}^{\infty} x_{2} f_{L}\left(y x_{2}\right) f_{v}\left(x_{2}\right) d x_{2} .
$$

Equation (15) represents the PDF of the quotient random variable $Y=X_{1} / X_{2}{ }^{\prime \prime}$

$$
\begin{gathered}
f_{L}\left(y x_{2}\right)=\frac{x_{2} y^{\alpha_{1}-1} e^{-x_{2} y / \beta_{1}}}{\Gamma\left(\alpha_{1}\right) \beta_{1}^{\alpha_{1}}}, \\
f_{v}\left(x_{2}\right)=\frac{x_{2}^{\alpha_{2}-1} e^{-x_{2} / \beta_{2}}}{\Gamma\left(\alpha_{2}\right) \beta_{2}^{\alpha_{2}}}, \\
h_{2}(y)=\int_{0}^{\infty} x_{2}\left(\frac{x_{2} y^{\alpha_{1}-1} e^{-x_{2} y / \beta_{1}}}{\Gamma\left(\alpha_{1}\right) \beta_{1}^{\alpha_{1}}}\right)\left(\frac{x_{2}^{\alpha_{2}-1} e^{-x_{2} / \beta_{2}}}{\Gamma\left(\alpha_{2}\right) \beta_{2}^{\alpha_{2}}}\right) d x_{2}
\end{gathered}
$$

make constant

$$
\begin{gathered}
C=\frac{y^{\alpha_{1}-1}}{\Gamma\left(\alpha_{1}\right) \Gamma\left(\alpha_{2}\right) \beta_{1}^{\alpha_{1}} \beta_{2}^{\alpha_{2}}}, \\
h_{2}(y)=C \int_{0}^{\infty} x_{2}^{\alpha_{1}+\alpha_{2}-1} e^{-x_{2}\left(y / \beta_{1}+1 / \beta_{2}\right)} d x_{2} .
\end{gathered}
$$

The solution to (18) provides the PDF of the residence time or the combined four-parameter gamma distribution RTD model noted as $E(t)$ in (19) as follows:

$$
\begin{array}{r}
E(t)=\frac{\Gamma\left(\alpha_{1}+\alpha_{2}\right)}{\Gamma\left(\alpha_{1}\right)\left(\alpha_{2}\right)}\left(\frac{\beta_{1}}{\beta_{2}}\right)^{\alpha_{2}}\left(\frac{t^{\alpha_{1}-1}}{\left(t+\beta_{1} / \beta_{2}\right)^{\alpha_{1}+\alpha_{2}}}\right), \\
\alpha_{1}, \alpha_{2}, \beta_{1}, \beta_{2} \geq 0
\end{array}
$$

taking the first moment of (19) about the mean using (3) gives

$$
t_{m}=\left(\frac{\beta_{1}}{\beta_{2}}\right)\left(\frac{\alpha_{1}}{\alpha_{2}-1}\right),
$$

where the mean travel distance is

$$
L=\alpha_{1} \beta_{1}
$$

and the mean travel linear velocity is

$$
v=\left(\alpha_{2}-1\right) \beta_{2} .
$$

Taking the second moment of (19) about the mean using (4) gives

$$
\sigma^{2}=\left(\frac{\beta_{1}}{\beta_{2}}\right)^{2}\left(\frac{\alpha_{1}}{\alpha_{2}-1}\right)\left(\frac{\alpha_{1}+1}{\alpha_{2}-2}-\frac{\alpha_{1}}{\alpha_{2}-1}\right) .
$$


The individual distributions of $L$ and $v$ provide insight into predicting the characteristics of the transformed distribution.

Assistance in deriving the intermediate steps between equations (18) and (19), between equations (19) and (20), and between equations (19) and (23) came from [53-56].

\section{Advection-Dispersion Equation RTD Model}

The one-parameter advection-dispersion equation RTD model is obtained from the dimensionless effluent tracer concentration in (24) which is derived from the solution to Danckwerts' "Open-Open System Boundary Conditions" and then applying (1) to (24) to produce $(25)[2,3]$ as

$$
\Psi(1, \theta)=\frac{C_{T}(L, t)}{C_{T_{0}}}=\frac{1}{2 \sqrt{\pi \theta / \mathrm{Pe}}} \exp \left[\frac{-(1-\theta)^{2}}{4 \theta / \mathrm{Pe}}\right] .
$$

Equation (24) is derived in [2, 3] as

$$
E(t)=\frac{\Psi(1, \theta)}{\int_{0}^{\infty} \psi d \theta}
$$

Equation (25) is modified from the $E(t)$ presented in [2, 7].

The Péclet number (Pe) in (24) is computed via (26) after both the mean residence time and the distribution variance are calculated

$$
\frac{\sigma^{2}}{t_{m}^{2}}=\frac{2}{\mathrm{Pe}}+\frac{8}{\mathrm{Pe}^{2}}
$$

Equation (26) is derived in [2] as

$$
t_{m}=\left(1+\frac{2}{\mathrm{Pe}}\right) \tau
$$

and solving (27) provides the space time $(\tau)[2]$.

\section{Laboratory Setup for the Validation of the Four-Parameter Gamma RTD Model}

A steady-state, non-ideal reactor of glass tubes was set up in the laboratory to simulate a plug-flow reactor (PFR). The glass tubing was borosilicate glass with an inner diameter of $0.4 \mathrm{~cm}$. The flow path model consisted of $1.4 \mathrm{~m}$ straight segments of glass tubing. The straight segments of glass tubing were connected by $180^{\circ}$ elbows made of Teflon tubing. The radius of each elbow was $0.079 \mathrm{~m}$ and the inner diameter of the Teflon tubing was $0.4 \mathrm{~cm}$. The linear length of the system was $32 \mathrm{~m}$. The system was calibrated such that flow rate in the system was maintained at $2.0-\mathrm{mL} / \mathrm{min}$. The injection mechanism to introduce the conservative tracer was a syringe delivering a volume of $5 \mathrm{~mL}$ for each trial. Two trials were conducted using $10 \mathrm{ppm}$ of the tracer dye rhodamine WT-20 and $10 \mathrm{ppm} \mathrm{Zn}$, zinc chloride $(\mathrm{ZnCl} 2)$. Discharge samples of the simulated PFR were collected at 20-minute intervals and analyzed using fluorometry and inductively coupled plasma optical emission spectrometry (ICP-OES) for rhodamine and zinc chloride, respectively. The rhodamine WT-20 tracer data was applied to the gamma and AdDE RTD models.

\section{Results and Discussion}

The results of the tracer study were used to develop the residence-time distribution (RTD) function. The RTD function $(E(t))$ for contaminant molecules in a non-ideal flow system is a probability density function (PDF) which can be interpreted to define the probability that contaminant particles present in the influent at time equals zero will arrive at the effluent after a time. The RTD is depicted as a plot of $E(t)$ versus time as time goes from zero to infinity (or a reasonably long time where the RTD approaches zero) [2$4,6-8]$.

$E(t)$ was determined by injecting a pulse of a conservative tracer (rhodamine WT-20) into the reactor, described in Section 4 , at time $(t)=0$ and then measuring the tracer concentration in the effluent as a function of time. The concentration and time data necessary for computing $E(t)$ was compiled in the Calc spreadsheet program of LibreOffice [57]. Using the Solver for Nonlinear Programming LibreOffice Calc extension [58], we computed the Péclet number (Pe) from (26) using the DEPS (Differential evolution and particle swarm optimization) algorithm [59].

Equation (28) represents the solution to the oneparameter advection-dispersion equation residence-time distribution function at time $\theta=0$. In this case the solution is infinity, although a finite tracer concentration should be expected for the initial time interval. Therefore, to compare the 3 RTD models, we disregard the RTD at time $=0$ as

$$
\begin{aligned}
\Psi(1, \theta=0) & =\frac{C_{T}(L, t)}{C_{T_{0}}} \\
& =\frac{1}{2 \sqrt{\pi \times 0 / \mathrm{Pe}}} \exp \left[\frac{-(1-0)^{2}}{4 \times 0 / \mathrm{Pe}}\right] \approx \infty .
\end{aligned}
$$

Equation (28) is derived in $[2,3]$ and is the same as (24) except that $\theta=0$ and

$$
E(t)=\frac{\Psi(1, \theta=0)}{\int_{0}^{\infty} \psi d \theta} \approx \infty .
$$

Equation (29) is modified from the $E(t)$ presented in $[2,7]$ and is the same as (25), except that $E(t)$ is shown to be approximately equal to $\infty$.

The normalized forms of the RTD for the tracer, the AdDE model, and the gamma model were computed in LibreOffice Calc. In order to determine the better RTD model, either the AdDE or the gamma, we had to calculate the mean-absolute deviation (MAD) [60] from the tracer RTD model using (30) as follows:

$$
\mathrm{MAD}=n^{-1} \sum_{i=1}^{n}\left|y_{i}-\widehat{y}_{i}\right|
$$

where $n$ represents the number of values where $y_{i}$ and $\hat{y}_{i}$ differ, $y_{i}$ is either the value of the AdDE or gamma RTD model, and $\widehat{y}_{i}$ is the value of the tracer RTD model [60].

The MAD associated with the gamma RTD model was approximately 0.16 while the MAD for the AdDE RTD model was approximately 0.26 . 


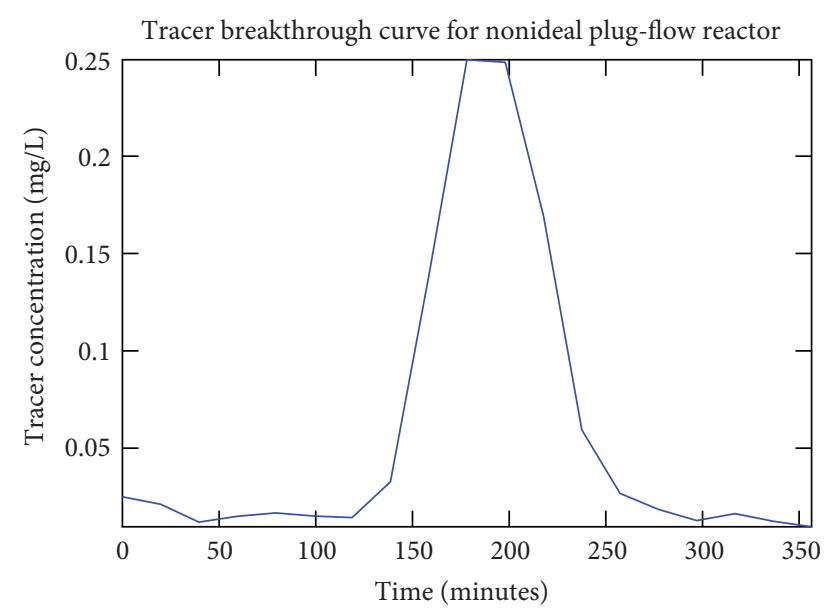

FiguRE 1: Tracer concentration versus time for the laboratory experiment. These data were used to determine the mean, variance, and Péclet number for the plug-flow reactor.

We used the DEPS algorithm to determine the four parameters $\left(\alpha_{1}, \beta_{1}, \alpha_{2}, \beta_{2}\right)$ to use in the gamma RTD model which provided a lower MAD than that produced from the AdDE model. Both a script and function files [61] were written in the M-file language of the numerical computation program GNU Octave [62]. GNU Octave uses either the FLTK toolkit [63] or gnuplot [64] to produce graphs. The following graphs in this paper were created using gnuplot rather than the FLTK toolkit. The script and function files used the four parameters for the gamma RTD model to solve equations (19)-(23) for the normalized gamma RTD model. The script and function files were also used to produce the graphs for the tracer breakthrough curve and the comparison of the normalized RTD curves. The graphical results of the laboratory, quantitative tracer study using rhodamine WT20 are shown in Figures 1 and 2.

Figure 1 shows the tracer breakthrough curve.

The results for the laboratory plug-flow reactor rhodamine dye study conducted are as follows The mean residence time $\left(t_{m}\right)$ is $\approx 186$ minutes which is from (3), the variance of the distribution $\left(\sigma^{2}\right)$ is $\approx 3276 \mathrm{~min}^{2}$ which is from (4), the dimensionless Péclet number is $\approx 25$ which is from (26), and the space time $(\tau)$ is $\approx 172$ minutes which is from (27).

To compare the 3 RTD models to each other (gamma from (19), advection-dispersion equation (AdDE) from (25), and tracer from (25)), we had to normalize each of the RTDs with dimensionless time.

Figure 2 shows the comparison of the three normalized RTD models.

The results for the normalized gamma RTD model's interpretation of the laboratory plug-flow reactor rhodamine dye study conducted using $\alpha_{1} \approx 50, \alpha_{2} \approx 50, \beta_{1} \approx 0.61$, and $\beta_{2} \approx 0.59$ are as follows.

The dimensionless mean residence time or mean time in the system $\left(t_{m}\right)$ is $\approx 1.07$ mean minutes which is from (20), the mean travel distance of tracer molecules is $\approx 32$ mean meters which is from (21), the mean travel linear velocity

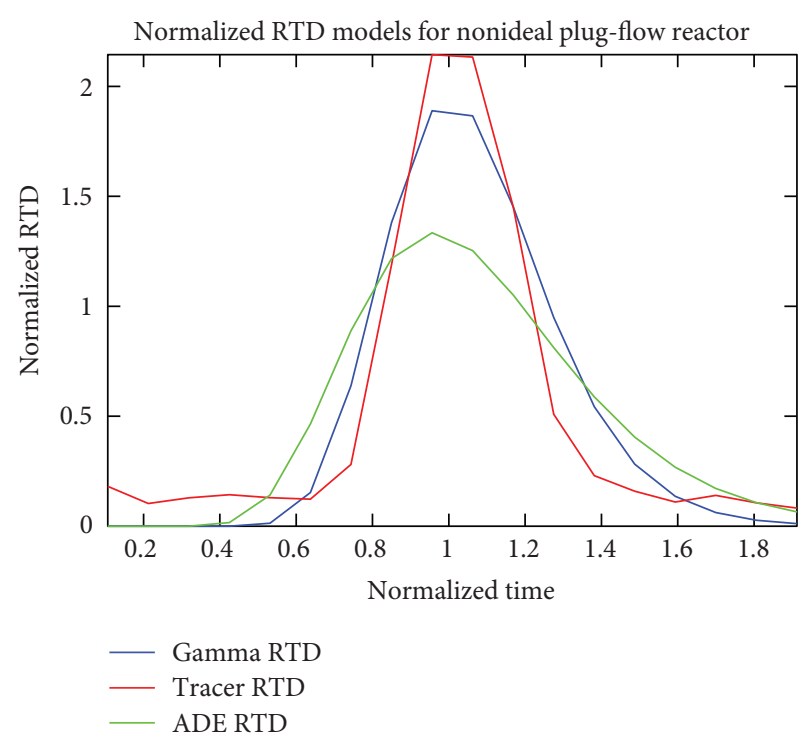

FIgURE 2: Comparing the normalized forms of the advectiondispersion equation RTD and the gamma RTD models to the normalized tracer RTD model for the laboratory tracer study.

(mean travel distance of tracer molecules/mean time in the system) is $\approx 30$ mean meters/minute which is from (22), and the dimensionless variance of the distribution $\left(\sigma^{2}\right)$ is $\approx 0.05$ mean min $^{2}$ which is from (23). The mean travel distance of $\approx 32$ mean meters is approximately equal to the straight-line horizontal distance of 32 meters for the laboratory reactor.

In an ideal plug-flow reactor (PFR) the apparent reactor velocity should be strongly correlated to the velocity of the peak of the tracer curve. This strong correlation is based on the shape of the velocity profile. In our study, the apparent reactor velocity (volumetric flow rate/area of the tube) is $\approx 9.55$ meters/hour compared to the velocity of the peak of the tracer curve (mean travel distance of tracer molecules/mean residence time), obtained from the gamma RTD model, of $\approx 9.77$ meters/hour. The velocity obtained from the gamma RTD model has a good correlation to the apparent reactor velocity with $\approx 2.3 \%$ error.

\section{Conclusions}

The normalized form of the gamma RTD function had a better fit with the tracer RTD function than the advectiondispersion equation RTD function. The mean-absolute deviation (MAD) from the normalized tracer RTD function for the normalized gamma RTD function was $\approx 0.16$ compared to $\approx 0.26$ for the normalized AdDE RTD model. The lower MAD value for the normalized gamma RTD function was also displayed visually in Figure 2.

As previously discussed in Section 5, the initial time value had to be removed from the comparison of the three normalized RTD models due to the normalized AdDE RTD function computing a value of $\infty$ at this time. This flaw presents a major setback in using the one-parameter advection-dispersion equation RTD function. 
In addition, the normalized gamma RTD function allows for the calculation of the mean travel linear velocity and the mean travel distance which are obtained from the $\alpha$ and $\beta$ parameters obtained from the best fit of the normalized gamma RTD to the normalized tracer RTD. The mean velocity and the mean distance traveled are tied back to the actual physical site due to the relation with time between the length and the linear velocity. This information is not available with the normalized AdDE RTD function.

For those reasons, we conclude that the jointly combined four-parameter gamma distribution RTD function better models the non-ideal flow present in the laboratory plugflow reactor than the one-parameter advection-dispersion equation RTD function. Thus, the gamma RTD function is a suitable alternative to the advection-dispersion equation RTD function for quantitative tracer studies of other nonideal flow systems.

\section{Abbreviations}

\begin{tabular}{|c|c|}
\hline$L:$ & Travel distance \\
\hline$v:$ & Velocity \\
\hline$t$ or $T:$ & Time \\
\hline$t_{m}:$ & Mean residence time \\
\hline$\sigma^{2}:$ & Distribution variance \\
\hline$\theta:$ & Dimensionless time \\
\hline$C(t):$ & Concentration over time \\
\hline$C_{T}:$ & Concentration at time $t$ \\
\hline$C_{T 0}:$ & Concentration at time $t=0$ \\
\hline$V:$ & Reactor or system volume \\
\hline$V_{\text {eq }}:$ & Equivalent volume \\
\hline$A_{\text {eq }}:$ & Equivalent area \\
\hline eq: & Equivalent \\
\hline Q: & Volumetric flow rate \\
\hline$\tau:$ & Space time \\
\hline irv: & Independent random variable \\
\hline RTD: & Residence-time distribution \\
\hline$E(t):$ & Residence-time distribution function \\
\hline TTD: & Transit time distribution \\
\hline DTD: & Detention time distribution \\
\hline HRTD: & Hydraulic residence-time distribution \\
\hline AdDE or ADE: & Advection-dispersion equation \\
\hline AxDE: & Axial dispersion equation \\
\hline ADM: & Axial dispersion model \\
\hline CDE: & Convection dispersion equation \\
\hline PDF: & Probability density function \\
\hline PFR: & Plug-flow reactor \\
\hline Pe: & Péclet number \\
\hline MAD: & Mean-absolute deviation \\
\hline DEPS: & $\begin{array}{l}\text { Differential evolution and particle } \\
\text { swarm optimization algorithm. }\end{array}$ \\
\hline
\end{tabular}

\section{Future Work}

We will compare the four-parameter gamma RTD function to the advection-dispersion equation RTD function for a quantitative dye study that was performed at Mammoth Cave National Park, Ky, USA.

\section{Acknowledgments}

The authors would like to acknowledge financial support from the United States Department of Education Title 3; United States Department of Energy (DOE) National Nuclear Security Administration (NNSA); United States Geological Survey (Tennessee Water Science Center); and Tennessee State University (TSU) College of Engineering and the Department of Civil and Environmental Engineering. The authors also would like to acknowledge the assistance provided by other software not cited in this paper: LibreOffice Writer for their document processing; the JabRef reference manager (http://jabref.sourceforge.net/) for managing their citation collection; Inkscape (http://inkscape.org/) for editing the gnuplot figures produced in the.svg format; and Trisquel GNU/Linux (http://trisquel.info/), which is the GNU/Linux operating system distribution, where all computer work was performed.

\section{References}

[1] R. B. MacMullin and M. Weber, "The theory of short-circuiting in continuous-flow mixing vessels in series and kinetics of chemical reactions in such systems," Transactions of American Institute of Chemical Engineers, vol. 31, no. 2, pp. 409-458, 1935.

[2] H. S. Fogler, Elements of Chemical Reaction Engineering, Prentice Hall PRT, Upper Saddle River, NJ, USA, 3rd edition, 1999.

[3] O. Levenspiel, Chemical Reaction Engineering, John Wiley \& Sons, New York, NY, USA, 3rd edition, 1999.

[4] P. V. Danckwerts, "Continuous flow systems. Distribution of residence times," Chemical Engineering Science, vol. 2, no. 1, pp. $1-13,1953$.

[5] R. Shinnar and P. Naor, "Residence time distributions in systems with internal reflux," Chemical Engineering Science, vol. 22, no. 10, pp. 1369-1381, 1967.

[6] C. G. Hill Jr., An Introduction to Chemical Engineering Kinetics \& Reactor Design, John Wiley \& Sons, New York, NY, USA, 1977.

[7] G. Tchobanoglous, F. L. Burton, H. D. Stensel, and Metcalf \& Eddy, Inc., Wastewater Engineering: Treatment and Reuse, McGraw-Hill, New York, NY, USA, 4th edition, 2003.

[8] L. D. Schmidt, The Engineering of Chemical Reactions, Oxford University Press, New York, NY, USA, 1998.

[9] V. K. Pareek, R. Sharma, C. Cooper, and A. Adesina, "Solids residence time distribution in a three-phase bubble column reactor: an artificial neural network analysis," The Open Chemical Engineering Journal, vol. 2, no. 1, pp. 73-78, 2008.

[10] J. Čermáková, F. Scargiali, N. Siyakatshana, V. Kudrna, A. Brucato, and V. MacHoň, "Axial dispersion model for solid flow in liquid suspension in system of two mixers in total recycle," Chemical Engineering Journal, vol. 117, no. 2, pp. 101-107, 2006.

[11] C. Laquerbe, J. C. Laborde, S. Soares et al., "Computer aided synthesis of RTD models to simulate the air flow distribution in ventilated rooms," Chemical Engineering Science, vol. 56, no. 20, pp. 5727-5738, 2001.

[12] S. J. Royaee and M. Sohrabi, "Comprehensive study on wastewater treatment using photo-impinging streams reactor: residence time distribution and reactor modeling," Industrial \& Engineering Chemistry Research, vol. 51, no. 11, pp. 4152-4160, 2012.

[13] J. C. Williams and M. A. Rahman, "The continuous mixing of particulate solids," Journal of the Society of Cosmetic Chemists, 
vol. 21, no. 1, pp. 3-36, 1970, http://journal.scconline.org/ abstracts/cc1970/cc021n01/p00003-p00036.html.

[14] C. G. C. C. Gutierrez, E. F. T. S. Dias, and J. A. W. Gut, "Investigation of the residence time distribution in a plate heat exchanger with series and parallel arrangements using a nonideal tracer detection technique," Applied Thermal Engineering, vol. 31, no. 10, pp. 1725-1733, 2011.

[15] C. G. C. C. Gutierrez, E. F. T. S. Dias, and J. A. W. Gut, "Residence time distribution in holding tubes using generalized convection model and numerical convolution for non-ideal tracer detection," Journal of Food Engineering, vol. 98, no. 2, pp. 248-256, 2010.

[16] A. P. Torres and F. A. R. Oliveira, "Residence time distribution studies in continuous thermal processing of liquid foods: a review," Journal of Food Engineering, vol. 36, no. 1, pp. 1-30, 1998.

[17] M. E. Rodrigues, A. R. Costa, M. Henriques, J. Azeredo, and R. Oliveira, "Wave characterization for mammalian cell culture: residence time distribution," New Biotechnology, vol. 29, no. 3, pp. 402-408, 2012.

[18] J. B. Bassingthwaighte, "Physiology and theory of tracer washout techniques for the estimation of myocardial blood flow: flow estimation from tracer washout," Progress in Cardiovascular Diseases, vol. 20, no. 3, pp. 165-189, 1977.

[19] J. N. Carleton, Modeling approaches for treatment Wetlands [Dissertation], University of Maryland, College Park, Md, USA, 2009, http://hdl.handle.net/1903/9585.

[20] J. N. Carleton and H. J. Montas, "A modeling approach for mixing and reaction in wetlands with continuously varying flow," Ecological Engineering, vol. 29, no. 1, pp. 33-44, 2007.

[21] J. N. Carleton and H. J. Montas, "An analysis of performance models for free water surface wetlands," Water Research, vol. 44, no. 12, pp. 3595-3606, 2010.

[22] Z. Q. Deng, H. S. Jung, and B. Ghimire, "Effect of channel size on solute residence time distributions in rivers," Advances in Water Resources, vol. 33, no. 9, pp. 1118-1127, 2010.

[23] R. A. Payn, M. N. Gooseff, D. A. Benson et al., "Comparison of instantaneous and constant-rate stream tracer experiments through non-parametric analysis of residence time distributions," Water Resources Research, vol. 44, no. 6, Article ID W06404, 10 pages, 2008.

[24] R. Painter, T. Byl, L. Sharpe, V. Watson, and T. Patterson, "A residence time distribution approach to biodegradation in fuel impacted karst aquifers," Journal of Civil \& Environmental Engineering, vol. 2, no. 5, article 121, 2012.

[25] R. Painter, T. Byl, L. Sharpe, A. Kheder, and J. Harris, "The role of attached and free-living bacteria in biodegradation in karst aquifers," Water, vol. 3, pp. 1139-1148, 2011.

[26] T. D. Byl and R. Painter, "Microbial adaptations to karst aquifers with contaminants," in Proceedings of the 19th Tennessee American Water Resources Association (AWRA), Tennessee Water Resources Symposium, pp. 2C-9-2C12, Burns, Tenn, USA, 2009.

[27] M. Martin and R. Painter, "Use of independent gamma distribution to describe tracer break-through curves," in Proceedings of the 19th Tennessee American Water Resources Association (AWRA), Tennessee Water Resources Symposium, pp. P10-PP10, Burns, Tenn, USA, 2009.

[28] I. Embry, V. Roland, R. Painter, R. Toomey, and L. Sharpe, "Quantitative dye tracing-development of a new interpretative method," in Proceedings of the 22nd Tennessee American Water Resources Association (AWRA), Tennessee Water Resources Symposium, pp. 1C-6-1C-16, Burns, Tenn, USA, 2012.
[29] R. H. Kadlec, "Effects of pollutant speciation in treatment wetlands design," Ecological Engineering, vol. 20, no. 1, pp. 1-16.

[30] M. Hrachowitz, C. Soulsby, D. Tetzlaff, I. A. Malcolm, and G. Schoups, "Gamma distribution models for transit time estimation in catchments: physical interpretation of parameters and implications for time-variant transit time assessment," Water Resources Research, vol. 46, no. 10, Article ID W10536, 15 pages, 2010.

[31] J. W. Kirchner, X. Feng, and C. Neal, "Catchment-scale advection and dispersion as a mechanism for fractal scaling in stream tracer concentrations," Journal of Hydrology, vol. 254, no. 1-4, pp. 82-101, 2001.

[32] J. W. Kirchner, D. Tetzlaff, and C. Soulsby, "Comparing chloride and water isotopes as hydrological tracers in two Scottish catchments," Hydrological Processes, vol. 24, no. 12, pp. 1631-1645, 2010.

[33] J. W. Kirchner, X. Feng, and C. Neal, "Fractal stream chemistry and its implications for contaminant transport in catchments," Nature, vol. 403, no. 6769, pp. 524-527, 2000.

[34] A. Brovelli, O. Carranza-Diaz, L. Rossi, and D. A. Barry, "Design methodology accounting for the effects of porous medium heterogeneity on hydraulic residence time and biodegradation in horizontal subsurface flow constructed wetlands," Ecological Engineering, vol. 37, no. 5, pp. 758-770, 2011.

[35] V. Zahraeifard and Z. Deng, "Hydraulic residence time computation for constructed wetland design," Ecological Engineering, vol. 37, no. 12, pp. 2087-2091, 2011.

[36] M. S. Field, “The QTRACER2 program for tracer breakthrough curve analysis for tracer tests in karst aquifers and other hydrologic systems," U. S. Environmental Protection Agency, Office of Research and Development, EPA/600/R02/001, 2002, http://oaspub.epa.gov/eims/eimscomm.getfile? p_download_id=36351.

[37] M. S. Field, "Efficient hydrologic tracer-test design for tracermass estimation and sample-collection frequency, 1. Method development," Environmental Geology, vol. 42, no. 7, pp. 827-838, 2002.

[38] B. Zhou, Y. Jiang, Q. Wang, and M. Shao, "Chloride transport in undisturbed soil columns of the Loess Plateau," African Journal of Agricultural Research, vol. 6, no. 20, pp. 4807-4815, 2011.

[39] M. Vanclooster, D. Mallants, J. Diels, and J. Feyen, “Determining local-scale solute transport parameters using time domain reflectometry (TDR)," Journal of Hydrology, vol. 148, no. 1-4, pp. 93-107, 1993.

[40] R. D. Markovic, "Probability functions of best fit to distributions of annual precipitation and runoff," Hydrology Papers no. 8, Colorado State University, Fort Collins, Colo, USA, 1965.

[41] I. Kotlarski, "On characterizing the gamma and the normal distribution," Pacific Journal of Mathematics, vol. 20, no. 1, pp. 69-76, 1967, http://projecteuclid.org/DPubS? service=UI\& version=1.0\&verb=Display\&handle=euclid.pjm/1102992970.

[42] A. Stuart and K. Ord, Kendall's Advanced Theory of Statistics: Volume 1: Distribution Theory, Oxford University Press, New York, NY, USA, 6th edition, 1994.

[43] K. V. Bury, Statistical Distributions in Engineering, Cambridge University Press, New York, NY, USA, 1999.

[44] N. T. Kottegoda and R. Rosso, Statistics, Probability, and Reliability for Civil and Environmental Engineers, McGraw-Hill, New York, NY, USA, 1997.

[45] B. Bobée and F. Ashkar, The Gamma Family and Derived Distributions Applied in Hydrology, Water Resources Publications, Littleton, Colo, USA, 1991. 
[46] H. A. Loáiciga, "Residence time, groundwater age, and solute output in steady-state groundwater systems," Advances in Water Resources, vol. 27, no. 7, pp. 681-688, 2004.

[47] S. K. Singh, "Simplified use of gamma-distribution/nash model for runoff modeling," Journal of Hydrologic Engineering, vol. 9, no. 3, 240243 pages, 2004.

[48] S. Yue, "A bivariate gamma distribution for use in multivariate flood frequency analysis," Hydrological Processes, vol. 15, no. 6, pp. 1033-1045, 2001.

[49] H. Aksoy, "Use of gamma distribution in hydrological analysis," Turkish Journal of Engineering and Environmental Sciences, vol. 24, no. 6, pp. 419-428, 2000.

[50] I. E. Amin and M. E. Campana, "A general lumped parameter model for the interpretation of tracer data and transit time calculation in hydrologic systems," Journal of Hydrology, vol. 179, no. 1-4, pp. 1-21, 1996.

[51] P. K. Bhunya, R. Berndtsson, C. S. P. Ojha, and S. K. Mishra, "Suitability of gamma, chi-square, weibull, and beta distributions as synthetic unit hydrographs," Journal of Hydrology, vol. 334, no. 1-2, pp. 28-38, 2007.

[52] M. D. Springer, The Algebra of Random Variables, John Wiley \& Sons, New York, NY, USA, 1979.

[53] G. A. Korn and T. M. Korn, Mathematical Handbook for Scientists and Engineers: Definitions, Theorems, and Formulas for Reference and Review, McGraw-Hill, New York, NY, USA, 2nd edition, 1968.

[54] S. M. Selby, CRC Standard Mathematical Tables, The Chemical Rubber, Cleveland, Ohio, USA, 18th edition, 1970.

[55] A. Jeffrey, Handbook of Mathematical Formulas and Integrals, Elsevier/Academic Press, New York, NY, USA, 3rd edition, 2004.

[56] D. Zwillinger, S. G. Krantz, and K. H. Rosen, Eds., CRC Standard Mathematical Tables and Formulae, CRC Press, New York, NY, USA, 30th edition, 1995.

[57] "LibreOffice Calc (Version 3.5.4.2) [computer software]," The Document Foundation, 2012, http://www.libreoffice.org/.

[58] "Solver for nonlinear programming/NLPSolver (Version 0.9beta-1) [computer software]," Sun Microsystems, Inc., 2009, http://extensions.services.openoffice.org/en/project/NLPSolver.

[59] Apache OpenOffice.org, "NLPSolver," 2012, http://wiki.services.openoffice.org/wiki/NLPSolver.

[60] C. J. Willmott, K. Matsuura, and S. M. Robeson, "Ambiguities inherent in sums-of-squares-based error statistics," Atmospheric Environment, vol. 43, no. 3, pp. 749-752, 2009.

[61] S. C. Chapra, Applied Numerical Methods with MATLAB for Engineers and Scientists, McGraw-Hill, New York, NY, USA, 2nd edition, 2008.

[62] J. W. Eaton et al., GNU Octave (Version 3.6.1) [computer software], 2012, http://www.octave.org.

[63] B. Spitzak et al., FLTK (Version 1.3) [computer software], 2011, http://www.fltk.org/index.php.

[64] T. Williams, C. Kelley et al., gnuplot (Version 4.4.4) [computer software], 2011, http://www.gnuplot.info. 

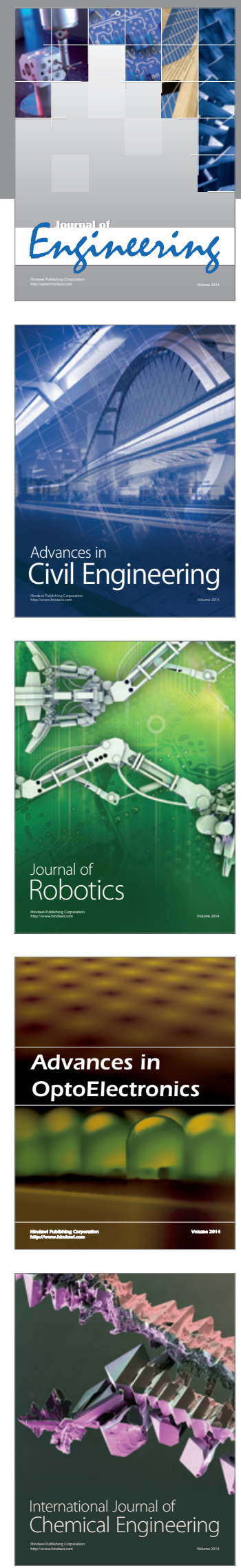

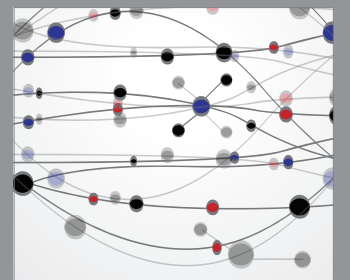

The Scientific World Journal
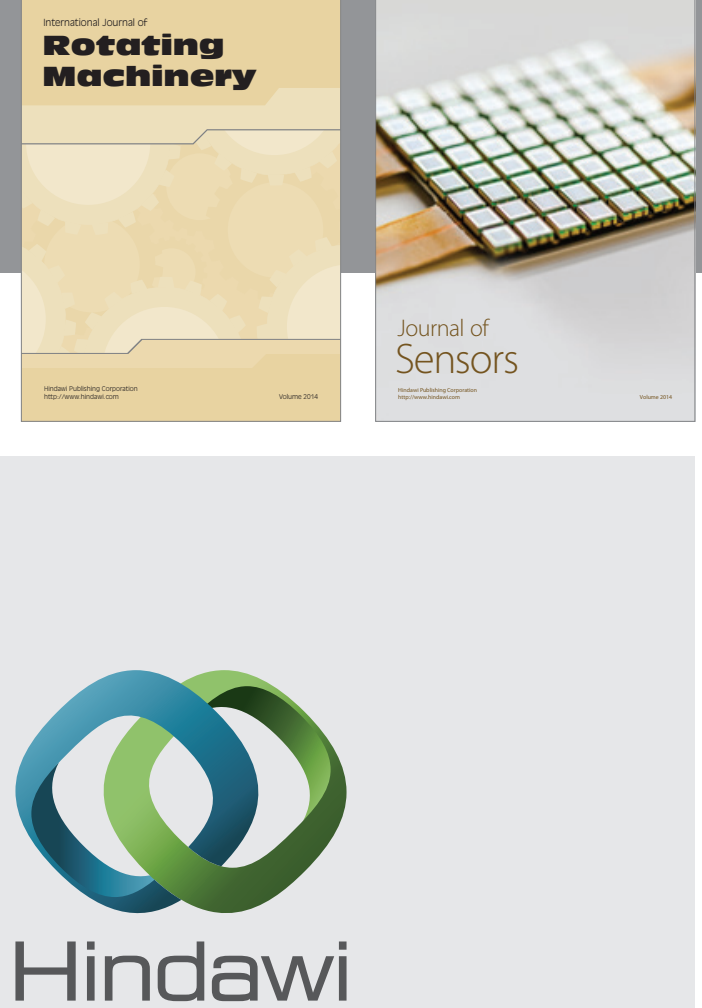

Submit your manuscripts at http://www.hindawi.com
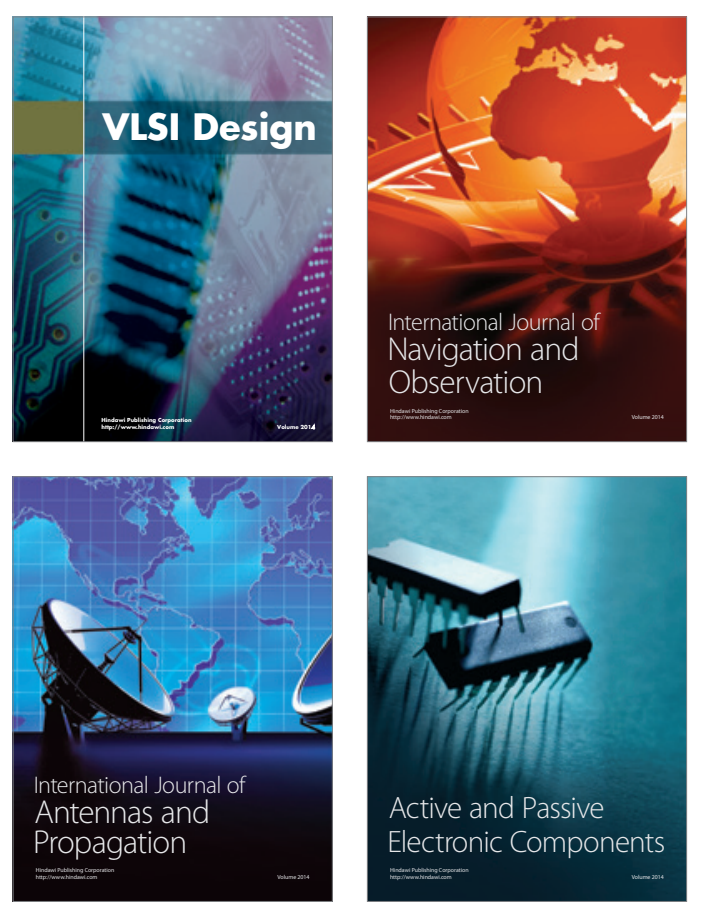
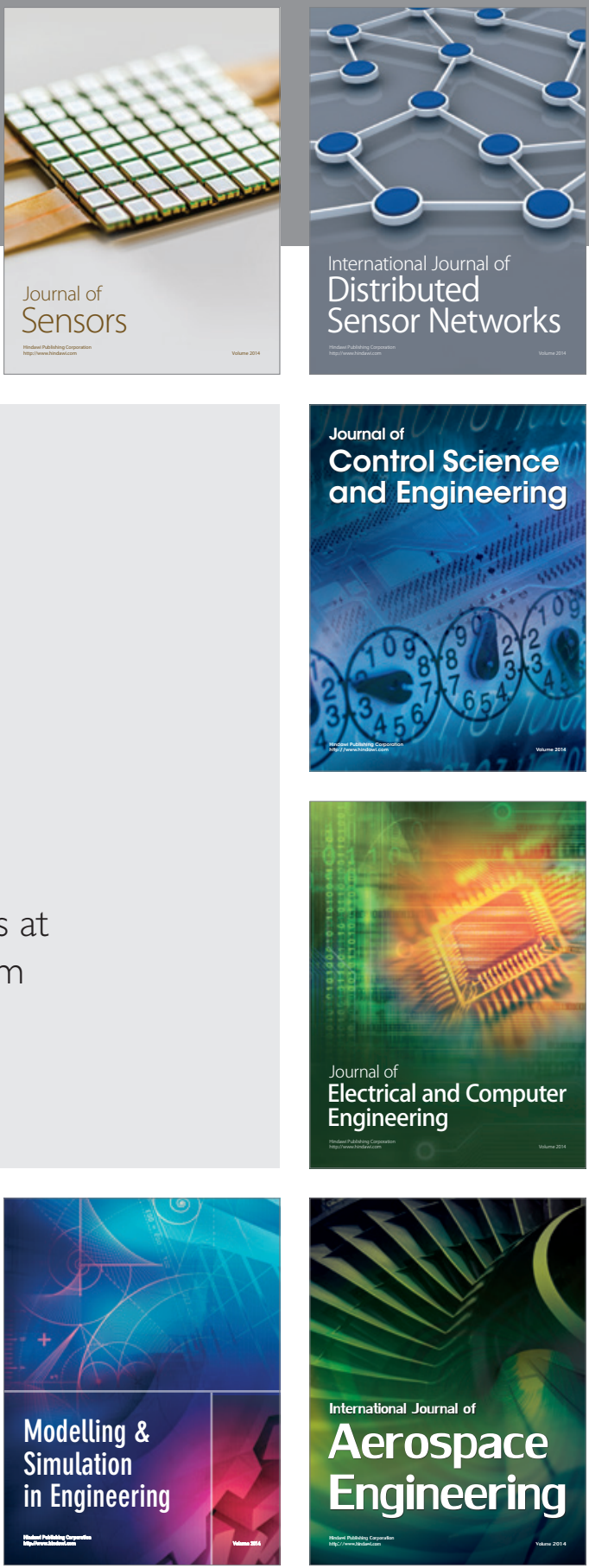

Journal of

Control Science

and Engineering
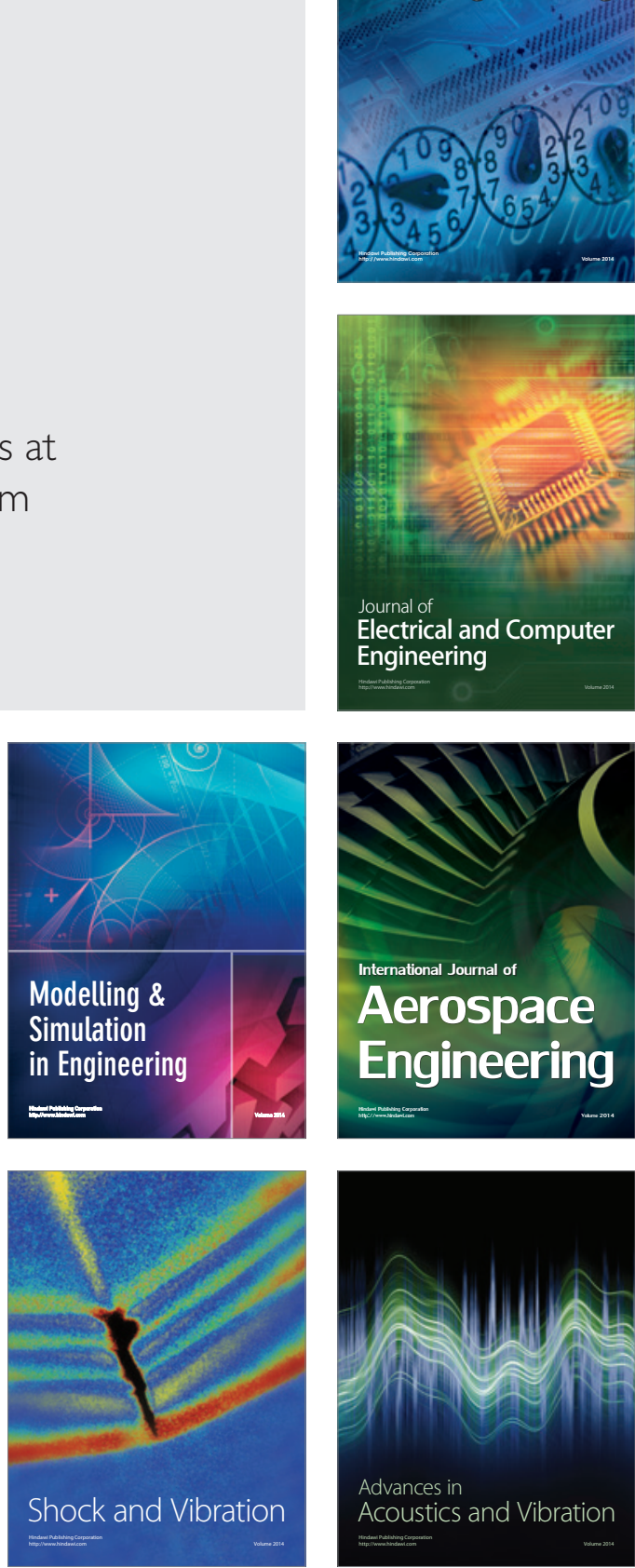